\title{
Specific humoral immunity in mink infected with the Aleutian disease virus (AMDV) and vaccinated against distemper
}

\author{
ANDRZEJ ŻMUDA, KRZYSZTOF KOSTRO, URSZULA LISIECKA \\ Department of Epizootiology and Clinic of Infectious Diseases, Faculty of Veterinary Medicine, \\ University of Life Sciences in Lublin, 20-612 Lublin, Głęboka 30, Poland
}

Żmuda A., Kostro K., Lisiecka U.

Specific humoral immunity in mink infected with the Aleutian disease virus (AMDV) and vaccinated against distemper

Summary

The Aleutian mink disease (AD) is one of the most serious threats to modern mink breeding. In farms where AD is endemic, a significant effect on breeding and reproductive effects is caused by permanent AMDV infection. The aim of this study was investigating the influence of subclinical infection of AMDV on the functional immune status and on the state of postvaccinal immunity against mink distemper. The analyses involved 30 brown mink females aged 12 weeks and allocated into an experimental group (group I) and control group (group II), with 15 animals in each. The minks from group I originated from the farm with an endemic form of Aleutian disease. The control minks from group II originated from the AMDV-uninfected farm. The dynamics of the growth of specific antibodies was examined after vaccination of the minks from both groups against distemper. Serum levels were determined in both groups with a seroneutralisation assay (SN). Obtaining positive values in the $\mathrm{SN}$ test in both groups of animals confirmed the induction of a specific immune response. There were significant differences in the magnitude of the positive $\mathrm{SN}$ assay values between the minks from group I and group II at all days of determination. The significantly lower SN values noted in the group minks with subclinical course of AMDV reveal that Aleutian disease can cause a suppressive effect on humoral response after vaccination.

Keywords: Aleutian mink disease, antibody levels, seroneutralization assay, vaccine immunity, distemper

Aleutian disease (AD) is a chronic and incurable disease of mink causing considerable economic losses in the breeding practice associated with low fecundity, recurrent miscarriages, high pup mortality rates in the first postnatal days, and many falls of several-monthold minks. A major impact on breeding and reproduction performance on mink farms with endemic $\mathrm{AD}$ is exerted by chronic AMDV infection $(4,5,7)$. Strong suppression of the immune system caused by chronic AMDV infection increases mink susceptibility to secondary bacterial infections, which are often an indirect cause a high rate of falls, in particular among young mink (7). The course of AMDV infections varies depending on the virulence of the infective strain, mink age, the functional status of the immune system, and the genotype of the animal (8). However, Aleutian disease in adult mink reared on Polish farms affected by its endemic form usually has a subclinical course (12). The growing problem of asymptomatic AMDV infections in mink on Polish breeding farms prompts the necessity of undertaking investigations aimed at elucidation of their effects on the functional immune status in chronically AMDV-infected mink and on post-vaccination immunity against various diseases acquired by the animals.

The aim of the study was to assess the effects of asymptomatic AMDV infections on specific humoral immunity in animals with subclinical AMDV infection vaccinated against distemper.

\section{Material and methods}

Experimental animals. The investigations were carried out on two mink farms comprising 45000 and 25000 females of the basic herd. The first herd with 45000 mink females was the experimental group. Approximately $60 \%$ of the mink reared on this farm were diagnosed with chronic subclinical AMDV infection over three rearing seasons. The infections were confirmed by counter current immunoelectrophoresis (CIEP) and additionally by Polymerase Chain Reaction (PCR). In turn, the other farm comprising 25000 
females from the basic herd, in which the AMDV infection was excluded with the use of the same diagnostic methods as on the first farm, served as the control group. The sanitary conditions prevailing on the farms were assessed as good. The minks were fed in accordance with standards recommended for this species; the animals received mineral and vitamin supplementation and had constant access to water. They were fed according to identical schemes on both farms, as the feed was prepared by the same feed kitchen.

The proper analyses involved 30 brown mink females aged 12 weeks and allocated into an experimental group (group I) and a control group (group II), with 15 animals in each. The mink from group I originated from the farm with the endemic form of Aleutian disease. The selection of the experimental animals was based on the presence of specific anti-AMDV antibodies detected with the CIEP method and the presence of viral genomic DNA in blood was confirmed with the use of PCR. In turn, the control mink from group II originated from the AMDV-uninfected farm. The mink from both groups were immunised against distemper, and the dynamics of the appearance of specific antibodies, whose serum levels were determined in both groups with a seroneutralisation assay at days $0,7,14,21$, and 28 after immunisation, was examined. The investigations mentioned above received consent of the II Local Ethics Committee for Experiments on Animals, University of Life Sciences in Lublin (Resolution 30/2010).

Serological diagnostics of AMDV. The biological material for the AMDV serological diagnostics included blood samples collected from capillaries intravitally by incision of claws of the females from group I and II. (CIEP), which is routinely used for the diagnosis of AD due to its high specificity and very high sensitivity, was used for the analyses (3).

Molecular diagnostics of AMDV. The biological material for the genetic tests included blood samples collected from incised claws of mink in which the presence of specific anti-AMDV antibodies had previously been detected with the use of CIEP. Blood was collected into test tubes containing $5 \mathrm{Mm}$ EDTA as an anticoagulant agent. Genomic DNA of the virus was isolated with a DNA isolation kit (DNA Isolation Kit for Mammalian Blood, Roche, Germany) following the manufacturer's instructions. The isolated DNA was amplified with the PCR method with the use of primers designed based on the AMD virus nucleotide sequences available in the GenBank database (NCBI). The NS1 gene was analysed due to the large representation of this gene in the database (13). To this end, PCR products that were localised on the gel as predicted during the electrophoresis process after comparison to the standard (DNA ladder) were sequenced and compared with the nucleotide sequences available in the GenBank database. During the analysis, the most optimal (conservative) fragments of the AMDV genome were typed as a site of annealing of amplification primers. Prior to the amplification, the reaction conditions were optimised by experimental determination of the most favourable concentration of polymerase, magnesium ions, deoxynucleotides (dNTP), and primers as well as the hybridisation temperature and the number of cycles. Next, the multiplex PCR method was compared with the seminested PCR technique in terms of their sensitivity and speci- ficity in the diagnosis of Aleutian disease. The semi-nested PCR method was demonstrated to be more specific than the multiplex method with the use of the selected primers.

Polymerase Chain Reaction (PCR). PCR was performed using a pair of primers for the NS1 gene from the AMDV genome. This non-structural gene is characterised by the presence of regions with low variability, therefore the primers intended for PCR amplification were selected from these regions using the BLAST program available on the NCBI website. The following temperature profile was adopted for the amplification reactions: $94^{\circ} \mathrm{C}, 5 \mathrm{~min}$ - initial denaturation of the matrix followed by 35 amplification cycles consisting of the following steps: $94^{\circ} \mathrm{C} 1 \mathrm{~min}$. - denaturation, $58^{\circ} \mathrm{C} 1 \mathrm{~min}$. - primer annealing, and $72^{\circ} \mathrm{C}$ 1 min. - DNA chain extension. The final DNA chain extension was performed at $72^{\circ} \mathrm{C}$ for $5 \mathrm{~min}$. The reaction mixture comprised $500 \mathrm{ng}$ of genomic DNA, $2.5 \mu 110 \times$ of concentrated PCR buffer $(100 \mu \mathrm{M}$ Tris-HCl, $\mathrm{pH} 8.8,500 \mu \mathrm{M}$ $\mathrm{KCl}), 1.5 \mathrm{mM} \mathrm{MgCl}_{2}, 50 \mu \mathrm{M} \mathrm{dNTP}, 0.5 \mu \mathrm{M}$ of each primer, and a 0.5 unit of Taq polymerase. The amplification reactions were carried out in a volume of $25 \mu \mathrm{l}$ using a UNO II thermocycler (Biometra, Germany).

Vaccine. A commercial vaccine called Distemink (United, Vaccines, Inc. Madison, WI USA 53719 U.S. Veterinary Licence No. 245), which is applied in the immunoprophylaxis of distemper, was used for the specific immunisation of the mink in groups I and II against the disease. The vaccine contains an attenuated distemper virus strain.

Determination of the serum level of specific antibodies against distemper. A seroneutralisation assay ( $\mathrm{SN}$ ) was used for detection of specific neutralising antibodies in the serum of mink immunised against distemper. The $\mathrm{SN}$ reaction was performed with the use of the beta method on Nunc Microplates (Thermo Scientific, Denmark) and increasing two-fold dilutions of the analysed sera which had been previously thermally inactivated at $56^{\circ} \mathrm{C} / 30 \mathrm{~min}$. Each serum dilution was mixed with a fixed dose of parvovirus $\left(100 \mathrm{CCID}_{50}\right)$ and kept at $4^{\circ} \mathrm{C}$ for $48 \mathrm{~h}$ and another $1 \mathrm{~h}$ at $37^{\circ} \mathrm{C}$. The mixture was used for inoculation of a CCC clone 81 continuous cell line in 8 wells per each serum dilution. The SN titre was established from the inverse of the highest dilution of the analysed serum inhibiting the cytopathic effect in 50\% of inoculated cell cultures. The serum level of specific neutralising antibodies was determined prior to administration of the vaccine (day 0 ) and next at days 7 , 14,21 , and 28 post inoculation.

\section{Results and discussion}

Results of serological assays. The dynamics of changes in the level of antibodies neutralising the distemper virus in the control mink immunised with the Distemink vaccine (group II) on the determination days is illustrated in Fig. 1. SN antibodies appeared in the blood of the immunised mink already at postvaccination day 7 and gradually increased over the subsequent periods. The highest titre of the SN antibodies throughout the observation period was detected at post-vaccination day 28 ; it was on average 512.0 \pm 16.0. The data presented in Figure 1 indicate that 


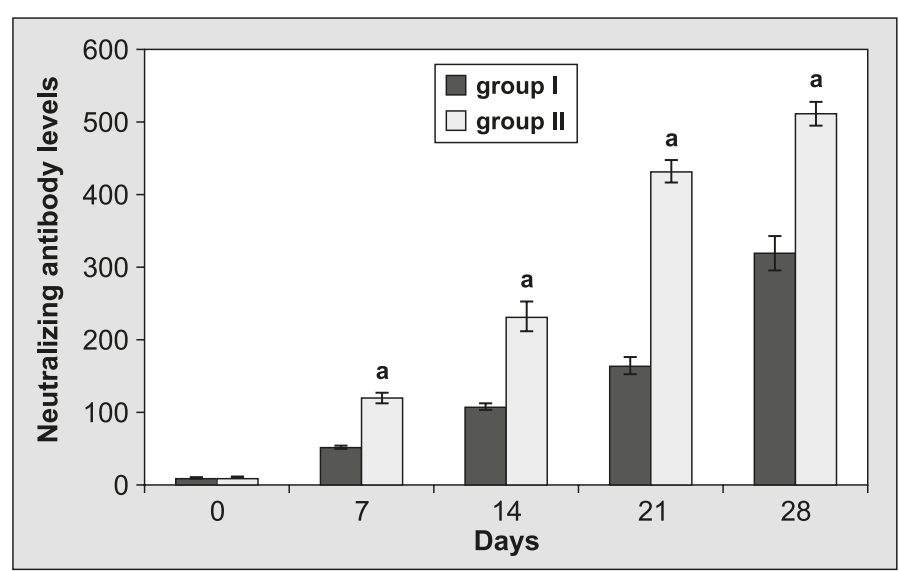

Fig. 1. Mean values of the serum level of neutralising antibodies in peripheral blood of minks with subclinical AMDV infection and control minks immunised against distemper $(\overline{\mathbf{x}} \pm \mathrm{SD})$

Explanations: group I - minks with subclinical AMDV infection; group II - AMDV uninfected minks; a - statistically significant difference between the groups at $\mathrm{P}<0.05$

the SN antibodies in the serum of the AMDV-infected mink (group I) immunised with the Distemink vaccine appeared at post-vaccination day 7 as well. It is noteworthy that the kinetics of the increase in the SN titre in the group I mink was identical to that in the animals from group II, but the mean values were significantly $(\mathrm{p} \leq 0.01)$ lower at all determination days.

Specific immunoprophylaxis in the case of infectious diseases of mink is targeted at reducing the susceptibility of animals to the diseases through the stimulation of specific immunity against a specific pathogen or a group of pathogens. Many factors influence the achievement of positive effects, i.e. immunity and protection from specific pathogens. The first group comprises factors that are directly related to the quality of vaccines, modes of vaccination, activity of some vaccine components, and suppressive effects of the antigenic components of vaccine viruses $(1,2,9-11)$. Another group of determinants exerting a significant impact on the effectiveness of vaccination is composed of factors that are closely related to the health status of the vaccinated animal. These include autoimmune diseases, immune deficiencies, disorders of the hormonal profile in the vaccinated animal, cancer diseases, infections caused by viruses with immunosuppressive effects on the vaccinated organism, nutrition, and conditions of maintenance of the vaccinated animal $(6,10,14)$.

The results of the present study indicate that the immune response in AMDV-infected mink involves suppression of the mechanisms of humoral immunity associated with the activity of B-cells, which was confirmed by the significantly lower mean values of the SN assay in the minks from group I in each analysed period, compared with the control animals (group II). In the present study, the specific immunological response in the experimental (group I) and control (group II) minks was assessed through determining the serum level of specific seroneutralising antibodies after the application of the Distemink vaccine, which is recommended for this animal species for immunoprophylaxis of specific distemper. The data demonstrate that the Distemink vaccine strongly stimulates specific humoral immune mechanisms, as confirmed by the high titres in seroneutralisation assays performed in the healthy minks (group II). The induction of a specific immune response in the distemper-immunised mink is indicated by the high titres of antibodies detected in the SN assay in the animals from group II. Therefore, the Distemink vaccine containing an attenuated distemper virus does not have suppressive activity towards immunocompetent cells; and so it does not reduce the reactivity of the immune system in minks. The development of protective (specific humoral) immunity against distemper after the administration of the Distemink vaccine required activation of a specific subpopulation of lymphocytes, which is determined by the properties of the strain contained in the vaccine. The induction of a specific immune response in the AMDV-infected minks immunised against distemper was also confirmed, as evidenced by the positive values of the SN assay (Fig. 1). There were significant differences in the magnitude of the positive SN assay values between the minks from group I and group II at all days of determination. The significantly lower SN values noted in the group-I minks reveal the mechanism of humoral immune response in the course of asymptomatic AMDV infection. It can be concluded that the specific humoral immunity is suppressed during chronic viral infections due to the persistent inflammation, which exerts an inhibitory effect on the specific humoral immune response after vaccination.

To sum up, it should be stressed that there is a close relationship between the functional status of the immune system and the level of acquired specific humoral immunity in mink with the subclinical form of Aleutian disease and vaccinated against distemper. The AMDV infection in mink, even with a subclinical course, can lead to the emergence of an immunological risk group that will not acquire sufficient immunity via the vaccination and will therefore require individual immunisation regimens to increase the effectiveness of the vaccine. It seems advisable to monitor the time of persistence of the titre value and the length of the protection period in minks with subclinical AMDV infection vaccinated against distemper to be able to confront these data with those provided by the vaccine producer.

\section{References}

1.Babiuk S., Baca-Estrada M. E., Foldvari M., Storms M., Rabussay D., Widera G., Babiuk L. A.: Electroporation improves the efficacy of DNA vaccines in large animals. Vaccine 2002, 20, 3399-3408.

2. Carpenter J. W., Appel M. J., Erickson R. C., Novilla M. N.: Fatal vaccineinduced canine distemper virus infection in black-footed ferrets. J. Am. Vet. Med. Assoc. 1976, 169, 961-964.

3.Dam-Tuxen R., Dahl J., Jensen T. H., Dam-Tuxen T., Struve T., Brunn L. Diagnosing Aleutian mink disease infection by a new fully automated ELISA 
or by counter current immunoelectophoresis: a comparison of sensitivity and specificity. J. Virol. Method 2014, 199, 53-60.

4. Hadlow W. J., Race R. E., Kennedy R. C.: Comparative pathogenicity of four strains of Aleutian disease virus for pastel and sapphire mink. Infect. Immun. 1983, 41, 1016-1023.

5. Kaaden O. R., Bartel E., Hass L., Kierek-Jaszczuk D., Löchelt M., Müller F. Neth R., Roth S., Stolze B., Dawen S. Van.: Mechanism contributing to the virus persistence in Aleutian disease. Dt. Tierärztl. Wschr. 1990, 97, 96-99.

6. Kehrli M. E., Burton J. L., Nonnecke B. J., Lee E. K.: Effects of stress on leukocyte trafficking and immune responses: implications for vaccination Adv. Vet. Med. 1999, 41, 61-81.

7. Kostro K., Wójcicka-Lorenowicz K., Siemionek J.: Choroba aleucka norek Med. Weter. 1999, 55, 432-429.

8. Kowalczyk M., JakubczakA., Horecka B., Kostro K.: A comparative molecular characterization of AMDV strains isolated from cases of clinical and subclinical infections. Vir. Gen. 2018, 54, 561-569.

9. Marhaug G., Hushyj G., Dowton S. B.: Serum amyloid A protein. Expression and primary structure based on cDNA sequences. J. Biol. Chem. 1990, 265 , $10049-10054$
10. Miyamoto T., Taura Y., Une S., Yoshitake M., Nakama S., Watanabe S.: Immunological responses after vaccination pre- and post-surgery in dogs. J. Vet. Med. Sci. 1995, 57, 29-32.

11. Mori S., Wolfinbanger J. B., Mivazawa M., Bloom M. E.: Replication of Aleutian mink disease parvovirus in lymphoid tissues of adult mink: involvement of follicular dendritic cells and macrophages. J. Virol. 1991, 65, 952-956.

12. Reichert M. Kostro $K$ : Effect of persistent infection of minks with Aleutian mink disease virus on reproductive failure. Bull. Vet. Inst. Pulawy 2014, 58, 369-373.

13. Reichert M., Kostro K.: NS1 gene based molecular characteristics of Aleutian mink disease virus circulating in Poland. Bull. Vet. Inst. Pulawy 2014, 58, 187-191.

14. Stolze B., Kaaden O. R.: Apparent lack of neutralizing antibodies in Aleutian disease due to masking of antigenic sites by phospholipids. Virology 1987, $158,174-180$.

Corresponding author: Andrzej Żmuda, DVM, PhD, Department of Epizootiology and Clinic of Infectious Diseases, Faculty of Veterinary Medicine, University of Life Sciences in Lublin, Glęboka 30, 20-612 Lublin, Poland; e-mail: andrzej.zmuda@up.lublin.pl 